\title{
Technologies for Cooling of Large Distributed Loads
}

\author{
J. R. Feller ${ }^{*}$ and L. J. Salerno ${ }^{\dagger}$ \\ NASA Ames Research Center, Moffett Field, CA, 94035, USA \\ A. Kashani ${ }^{\ddagger}$, B. P. M. Helvensteijn ${ }^{\S}$, and J. R. Maddocks ${ }^{* *}$ \\ Atlas Scientific, San Jose, CA, 95120, USA \\ G. F. Nellis ${ }^{\dagger \dagger}$ \\ University of Wisconsin-Madison, Madison, WI, 53706, USA \\ and \\ Y. B. Gianchandani \\ University of Michigan-Ann Arbor, Ann Arbor, MI, 48109, USA
}

\begin{abstract}
In future space applications, large and distributed loads will require active thermal control if their lifetimes are to be extended beyond one or two years. Examples include Zero Boil-Off (ZBO) cryogenic systems for exploration missions, and cooling of widely distributed sensor arrays and large deployable structures, such as mirrors and sunshades, for space science missions. These applications will require efficient means of heat transfer from extended structures or from several discrete elements to one or more remotely located heat rejection packages consisting of active and passive components. More or less stringent temperature control will also be required. We have recently undertaken a program to develop a number of technologies relevant to the issues associated with distributed cooling. These include circulation networks that transfer heat via steady flows of cold pressurized gas; gas rectifiers for use with linear pressure wave generators; and MEMS-based throttling valves for precise temperature control. This paper describes the studies that are underway to establish the performance potential of each.
\end{abstract}

\section{Nomenclature}

$c_{p} \quad=$ mass-specific heat at constant pressure

$D=$ tubing inner diameter

$f \quad=$ friction factor

$L \quad=$ heat transfer length

$\dot{m} \quad=$ mass flow rate

$P \quad=$ gas pressure

$\dot{Q}=$ heat transfer rate

$\dot{q}=$ heat flux (heat transfer rate per unit area)

$\dot{q}_{\lambda} \quad=$ heat transfer rate per unit length

\footnotetext{
${ }^{*}$ Research Scientist, Cryogenics Group, Sensors and Instrumentation Branch, Mail Stop 244-10, Moffett Field, CA.

${ }^{\dagger}$ Cryogenics Group Lead, Sensors and Instrumentation Branch, Mail Stop 244-10, Moffett Field, CA.

$\$$ President and Chief Scientist, Atlas Scientific, San Jose, CA.

$\S$ Research Scientist, Atlas Scientific, San Jose, CA.

${ }^{* *}$ Research Scientist, Atlas Scientific, UWCEG, Madison, WI.

${ }^{\dagger}$ Professor, University of Wisconsin Cryogenic Engineering Group, Department of Mechanical Engineering, Madison, WI.

\$ Professor. University of Michigan Solid State Electronics Lab, Ann Arbor, MI. 


$\begin{array}{ll}T & =\text { absolute temperature } \\ u & =\text { gas velocity } \\ \kappa & =\text { thermal conductivity } \\ \rho & =\text { gas density }\end{array}$

\section{Introduction}

$\mathrm{T}$

HE work described below was initiated in response to proposed space science missions that would require more or less precise thermal control of large structures (e.g., mirrors) or of widely separated discrete elements (e.g., sensor arrays). A collaboration among NASA Ames Research Center's Cryogenics Group, Atlas Scientific (based in San Jose, CA), the University of Wisconsin Cryogenic Engineering Group (UWCEG) and the University of Michigan Solid State Electronics Laboratory (UMSSEL) was formed to investigate the feasibility of using small, low-actuation-power, variable Joule-Thomson (J-T) expanders, fabricated using MEMS techniques, to provide a modest but minutely controllable cooling capacity to any number of points within a large distributed thermal load. Initial studies suggested, however, that a more efficient and versatile approach to the problem was to employ the MEMS devices as throttle valves in a network of circulation loops consisting of small diameter tubing within which a steady flow of pressurized pre-cooled helium gas transports heat from the distributed load to one or more remotely located cryocooler cold heads. Helium gas was chosen as the working fluid because of its high specific heat, its near-ideal gas behavior (above $50 \mathrm{~K}$ or so), and other desirable properties. In this scheme, heat is transported via the enthalpy flow $\dot{m} c_{p} T$, where $\dot{m}$ is the mass flow rate, $c_{p}$ the (nearly constant) mass-specific heat, and $T$ the gas temperature. The micro-valves then effect thermal control by limiting flow to given branches, or loops, of the circulation network.

This work was further motivated by NASA's recurring interest in enabling technologies for long-term storage of cryogenic propellants. Depending on mission requirements, reduced boil-off (RBO) or zero boil-off (ZBO) storage would likely require the use of advanced passive thermal control techniques, such as high performance multi-layer insulation (MLI). But in many cases (for example, multi-month loiters in low earth orbit) integration of active cooling components might also be necessary, or at least advantageous. For active RBO or ZBO preservation of liquid oxygen (LO2) or liquid methane (LCH4), this does not present a serious problem, at least not conceptually, as flight-like pulse tube refrigerators and other types of cryocoolers, operating at cold head temperatures of $90 \mathrm{~K}$ and above, and with sufficient cooling capacities (tens of Watts thermal), are available.

The same cannot be said for liquid hydrogen (LH2). Because high capacity $20 \mathrm{~K}$ cryocooler technology is presently, and for the foreseeable future will remain at such a low readiness level, direct cooling of LH2 tanks is not a feasible option. However, simple calculations indicate that, along with effective MLI, an actively cooled thermal radiation shield, maintained at $\sim 100 \mathrm{~K}$, is capable of nearly eliminating the radiative heat leak to $20 \mathrm{~K}$. Heat is thus intercepted and removed at the shield temperature using existing cryocooler technology. As discussed in the following section, system mass minimization requirements pointed to the application of distributed cooling.

\section{Overview of Passive and Active Heat Transfer Options}

A partial illustration of the evolution of distributed cooling concepts, as applied to active RBO of cryogenic propellants, is given in Fig. 1. Various cryocooler integration schemes were proposed and examined, some in more detail than others. Each option can be listed under one of two broad categories: (1) Point cooling (Fig. 1 (a), (b), and (c)), wherein the cryocooler cold head is integrated more or less directly with the cryogen (e.g., with a tank mixer), tank, or tank thermal shield; or (2) distributed cooling (Fig. 1 (d), (e), and (f)), wherein heat is transferred, by some means other than conduction, to the cold head from remote points or areas of a potentially very large structure (in this case the tank wall, thermal shield, or tank penetrations). The simplicity of option (a), direct integration with the tank mixer, is its main advantage. However, when the cooler is powered down, the cold finger represents a sizeable heat leak into the tank. This is addressed by option (b), wherein the cold head is isolated from the tank by a thermal switch, such as a heat pipe diode. Neither of these options are able to intercept the conduction heat leak through the tank support structure and other penetrations. More importantly, however, is that neither option, as stated above, is at this time applicable to LH2 storage. This leads to option (c), which provides an actively cooled thermal radiation shield, allowing heat interception at some temperature intermediate between that of the tank and of the thermal environment. In this way, most of the incident thermal radiation, as well as a significant portion of the conduction heat leak, can be prevented from reaching the tank wall. If the tank is at LO2 temperatures or above, a second cold stage at the tank temperature could be provided to lift the remaining heat leak. Further investigation, however, shows that in order to achieve a reasonable temperature uniformity, high thermal conduction rates would be 


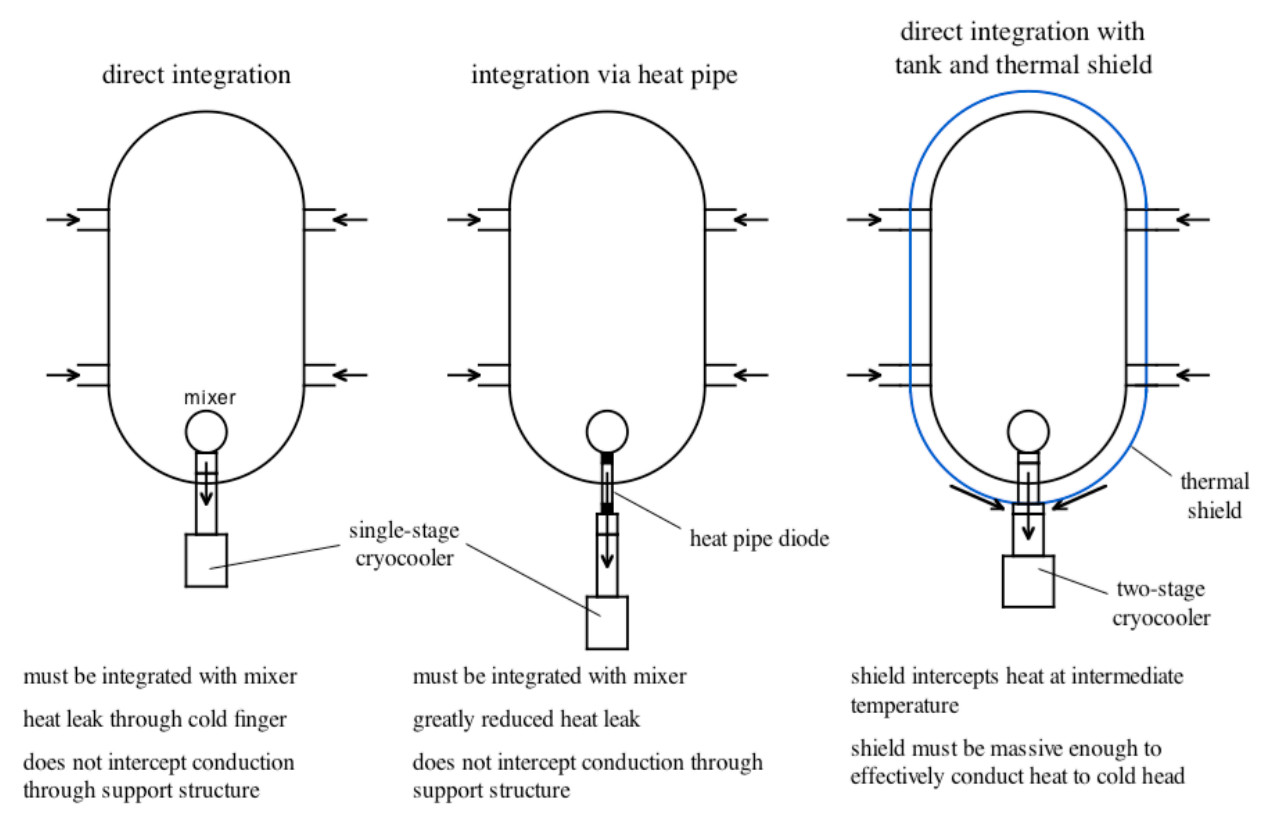

a)

b)

c)

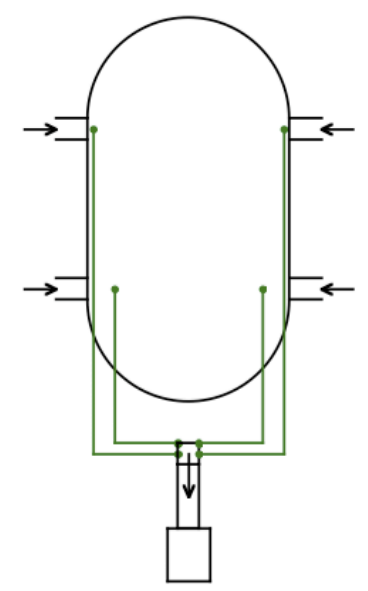

point (discrete) cooling using heat pipes or capillary pumped loops

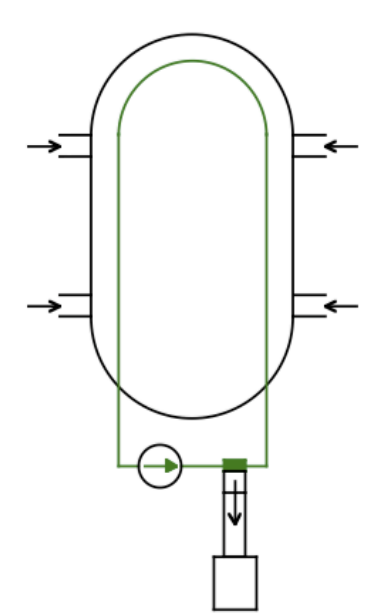

broad area cooling (BAC) using warm or cold circulator

heat removed by enthalpy flow of circulating gas

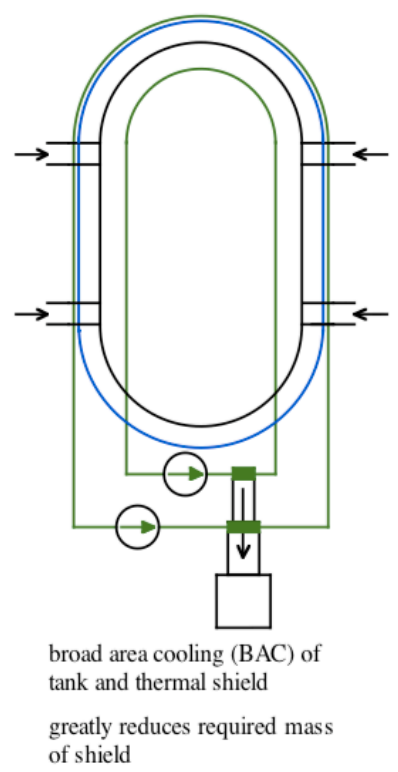

e)

f)

Figure 1. Some options for integration of a cryocooler cold head with a cryogenic propellant tank, with major advantages and disadvantages.

required, leading to a prohibitively massive shield ${ }^{1}$. In general, thermal coupling via conduction is not an attractive option for large or widely separated loads. For example, a thermal link composed of oxygen-free, high-conductivity (OFHC) copper capable of transporting one Watt over one meter with a temperature rise of $2.5 \mathrm{~K}$ would require a cross-sectional area of about $8.7 \mathrm{~cm}^{2}$, and would have a mass of $7.8 \mathrm{~kg}$. In addition to this mass penalty, the use of a conductive link limits the mechanical and electrical flexibility of the interface; it is difficult to ensure mechanical or electrical isolation without simultaneously reducing the thermal conductance.

The mass of the thermal shield and of the thermal interfaces to the tank penetrations can be dramatically reduced if distributed cooling techniques are employed. Heat pipes or capillary pumped loops (Fig. 1 (d)) are capable of passively conveying heat with very high effective conductivities. They are, however, essentially point-to-point heat 
transfer devices, and as such are more appropriately applied to the thermal management of arrays of compact structures. A promising recourse (Fig. 1 (e) and (f)) is the actively cooled circulation loop that was briefly described in the introduction. When thermally bonded to the shield, the network tubing itself serves as a continuous heat exchanger. This scheme is referred to as broad area cooling, or BAC. A BAC thermal radiation shield can in principle be remarkably light. With moderate flow rates through multiple parallel loops, a temperature uniformity of a few degrees is attainable using a very thin metal foil shield.

\section{The Actively Cooled Circulation Loop}

A schematic diagram of a basic BAC network is pictured in Fig. 2. In this case, the gas circulator is warm; it is partially isolated from the cold section of the network by a recuperator (depicted as a counter-flow heat exchanger). Heat is removed from the circuit via a heat exchanger that is directly integrated with a single discrete cold head. The BAC network would typically consist of a number of identical parallel cooling lines, distributed uniformly over the shield surface. As discussed in a previous paper ${ }^{2}$, the relations describing the temperature rise $\Delta T$ and pressure drop $\Delta P$ between the network inlet and outlet in terms of the mass flow rate $\dot{m}$ and the total heat load $\dot{Q}$ are simple and intuitive, provided certain conditions are met, as they usually are in most applications of interest. For a single cooling line of length $L$,

$$
\dot{Q}=\dot{q}_{\lambda} L=\dot{m} c_{p} \Delta T,
$$

where $\dot{q}_{\lambda}$ is the (assumed constant) heat load per unit length. Usually, $\Delta T$ is treated as a design parameter (i.e., it is the maximum allowable temperature rise). In that case, Eq. (1) states that the required mass flow rate is directly proportional to the total heat load on the line. The pressure drop is given by

$$
\Delta P=-\frac{1}{2} \frac{L}{D} f \rho u^{2}=-\frac{8 L}{\pi^{2} D^{5}} \frac{f}{\rho_{a v}} \dot{m}^{2},
$$

where $\rho$ is the gas density, $\rho_{a v}$ the average density between inlet and outlet, $u$ is the gas velocity, $f$ is the appropriate friction factor, and $D$ is the inner diameter of the cylindrical tube.

As stated above, high capacity $90 \mathrm{~K}$ pulse tube refrigerators are available at high technology readiness level (TRL). Likewise, high TRL flight-like gas circulators (compressors) exist and are used in reverse-Brayton and other recuperative-cycle cryocoolers. An attractive circulator option is to employ a small linear pressure wave generator, which is efficient and reliable, in conjunction with a flow rectifier. The rectifier consists of two opposed check valves, acting as flow diodes, and two small buffer volumes, acting as acoustic compliances. This is the fluiddynamic analog of the electrical AC-to-DC full-wave rectifier.

The recuperator is also a high TRL component. But it typically carries with it a substantial mass penalty. Ideally it should be as effective as possible, as any ineffectiveness will translate into an extra heat load on the cold head, with attendant increases in cryocooler mass and power requirements. On the other hand, the recuperator mass necessarily increases dramatically as its effectiveness is pushed toward 100 percent. The recuperator therefore represents a difficult design trade. 
The function of the recuperator is to thermally isolate the warm circulator from the cold flow loop. It would therefore be far preferable to employ a cold circulator. A promising candidate is the hybrid pulse tube / circulator $^{3}$ (Fig. 3), wherein the cold head is tapped into and a relatively small oscillating flow is diverted to a cold rectifying interface. The warm compressor is now thermally isolated by the regenerative heat exchanger, instead of the much more massive recuperator. Moreover, there is no need for the cold head heat exchanger, as shown in Fig. 2, nor for a second compressor to drive the circulation. In the entire system there are thus only four moving parts: two flexured pistons (dual opposed) in the linear compressor, and two check valves. The check valves, however, must be capable of reliably operating at cryogenic temperatures. Such systems, including reliable cryogenic check valves, have been developed and are used almost routinely at $\mathrm{UWCEG}^{4}$.

Of course, there are alternatives to the pulse tube refrigerator. For example, a turbo-Brayton, similar to the very successful unit developed by Creare ${ }^{5}$, which is currently in operation on the Hubble Space Telescope, might be considered a more natural choice for distributed cooling applications. It has, as it were, an intrinsic cold circulation loop that could be integrated directly with a distributed cooling network. This is assuming that it is capable of developing a flow rate compatible with the thermal and pressure constraints of the network, as given by Eqs. (1) and (2).

\section{Predicted Performance of a BAC Thermal Shield}

An illustration of the BAC concept is provided by an analysis that was performed by the NASA Ames Research Center (ARC) Cryogenics Group in support of the In-Space Cryogenic Propellant Depot (ISCPD) Project (funded by the NASA Headquarters Exploration System Research and Technology Division of the Office of Exploration Systems). The goal of the project was to develop a test bed for the evaluation of novel cryogenic fluid management (CFM) techniques, and to simulate (as closely as is possible in a 1-g environment and within the confines of a large but still limited vacuum chamber) the operational characteristics of an actual in-space cryogenic propellant storage facility. Had it been funded to completion, the test bed would have consisted of a 130 cubic foot capacity cryogen tank outfitted with various CFM components related to liquid acquisition, mass gauging, etc. The ARC role was to develop a ZBO demonstration, including an actively cooled lowmass thermal shield ${ }^{6}$. A diagram of the proposed $\mathrm{ZBO}$ system is shown in Fig. 4. The circuit consisting of the BAC lines, supply and return plumbing, recuperator ("CFHX"), cryocooler ("cryocooler 1"), and gas circulator corresponds to the schematic of Fig. 2.
Figure 4. ZBO concept for the ISCPD test bed. Thermal control elements not represented: Tank MLI blankets and the thermal shield (bonded to the BAC lines).

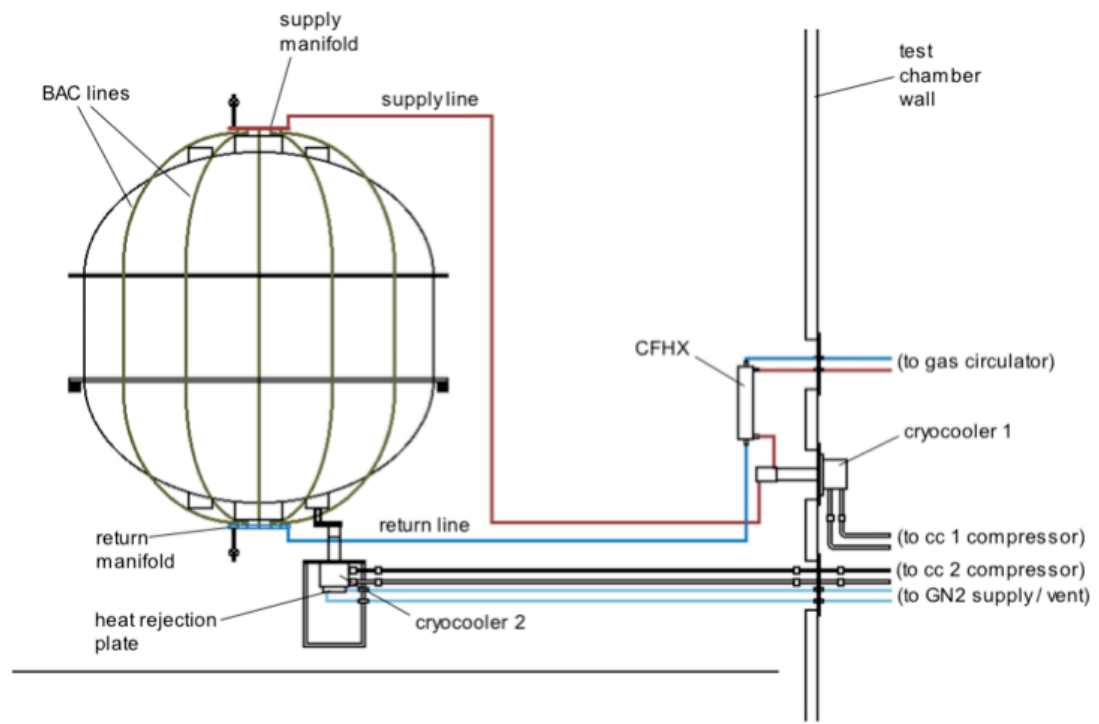




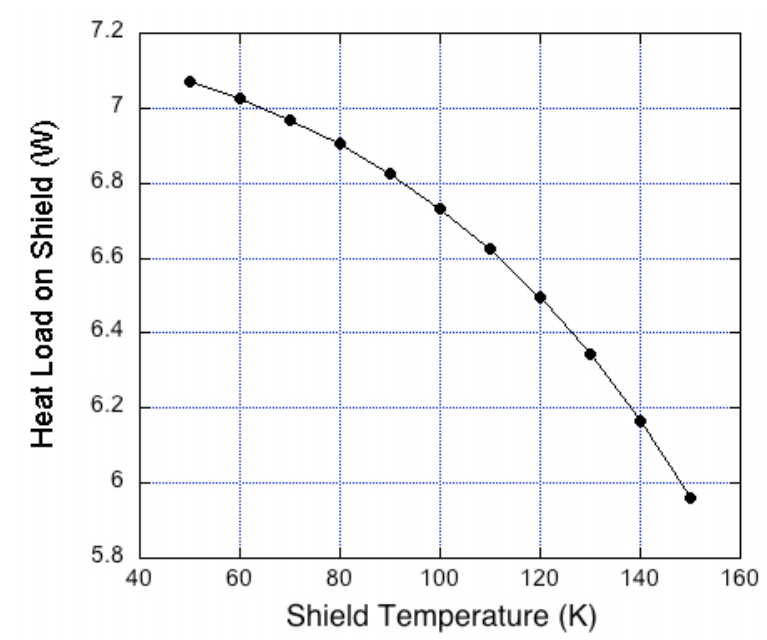

Figure 5. Net heat load on the shield as a function of shield temperature.

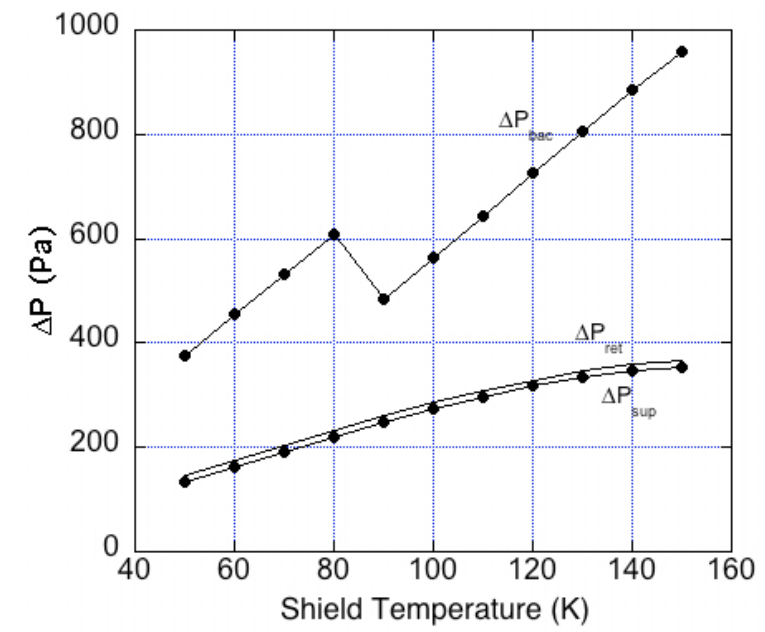

Figure 7. Pressure drops in the BAC network as functions of shield temperature. A turbulent-tolaminar transition is evident.

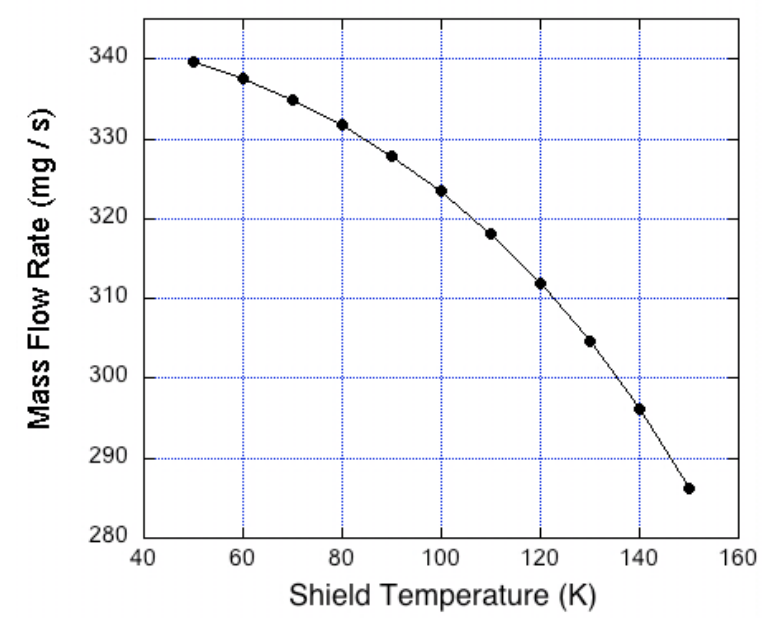

Figure 6. Required total mass flow rate as a function of shield temperature.

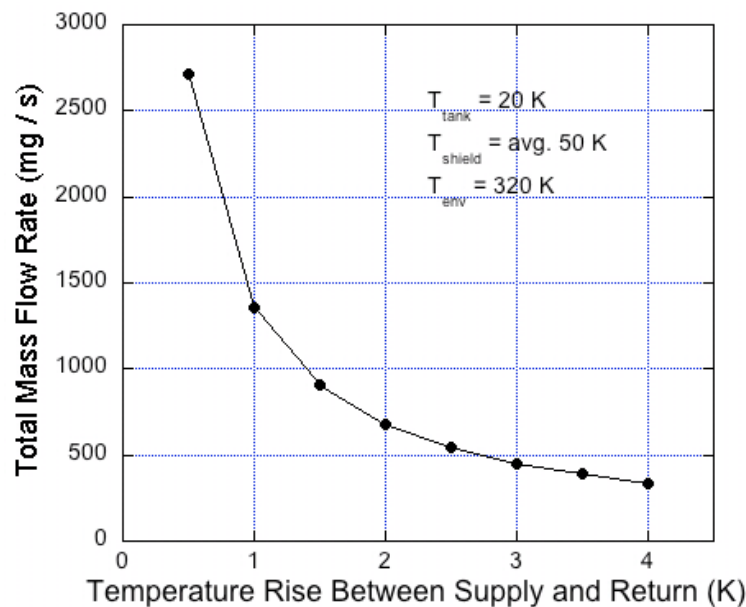

Figure 8. Total required mass flow rate as a function of imposed maximum inlet-to-outlet temperature rise.

Not shown are the MLI blankets surrounding the tank and the shield, and the metal foil shield itself.

Given the physical dimensions of the tank, the surface area of the actively cooled shield is estimated to be $12 \mathrm{~m}^{2}$. Assuming reasonably good passive shielding, the worst case (tank at $20 \mathrm{~K}$; environment at $320 \mathrm{~K}$, imposed by a variable-temperature enclosure) net heat load on the shield is around $10 \mathrm{~W}$.

The configuration of the BAC network is very simple: a number, $N_{B A C}=16$, of identical lines (cylindrical tubes, inner diameter $D=1 / 16$ in.) are connected in parallel, both geometrically and fluid-dynamically, to the supply and return plena, which are just low impedance tubes or chambers that communicate with the rest of the circulation loop. The lines are uniformly spaced; the separation distance, along the circumference of the shield, is $w_{B A C}$. The foil shield is bonded to the BAC lines using low temperature epoxy.

The shield temperature has a maximum midway between adjacent lines. This is found to be

$$
T_{M A X}=T_{0}+\frac{w_{B A C}{ }^{2}}{8 \kappa t} \dot{q},
$$


where $T_{0}$ is the shield temperature directly beneath the epoxy bonds, $\kappa$ is the thermal conductivity of the shield material (1100 Aluminum), $t \sim 2$ mil is the shield thickness, and $\dot{q}$ is the constant heat flux (net heat load on the shield per unit area). Because of the finite effective thermal conduction through the epoxy bond, the tube wall, and into the bulk of the gas stream, the temperature of the gas $T_{\text {gas }}$ will be lower than the shield temperature $T_{0}$. The difference is given by

$$
T_{0}-T_{\text {gas }}=R_{e f f} \dot{q}_{\lambda}=R_{e f f} \dot{q} w_{B A C} .
$$

The effective thermal resistance $R_{\text {eff }}$ depends on the thermal conductivity of the epoxy bond $(\sim 0.3 \mathrm{~W} / \mathrm{m} \cdot \mathrm{K})$, the bond's effective thickness and width, the thermal conductivity of the tube material, the tube diameter and wall thickness, and on the convective heat transfer coefficient that determines the effective thermal resistance between the tube wall and the gas stream. It is found, both theoretically and experimentally ${ }^{7}$, that $R_{\text {eff }}$ is dominated by the convective heat transfer.

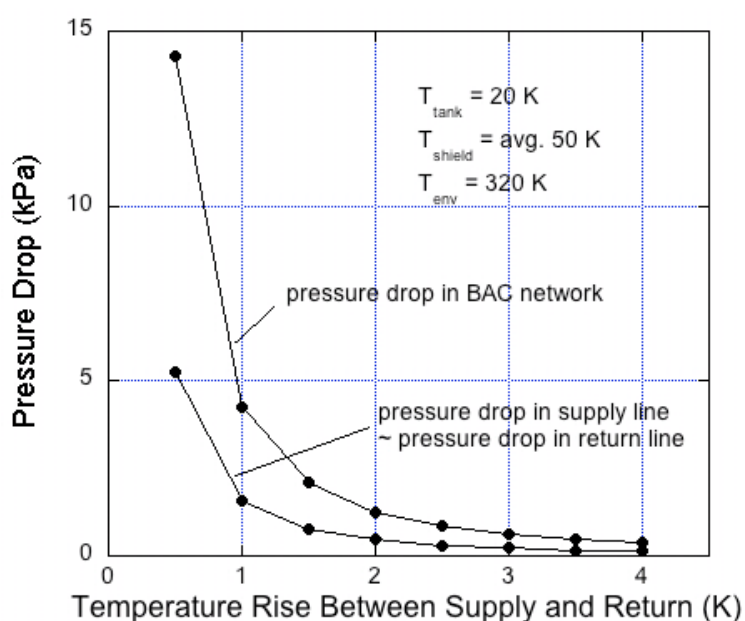

Figure 9. Pressure drops in the BAC network as functions of imposed maximum inlet-to-outlet temperature rise.

In most cases, the maximum temperature rise $T_{\text {MAX }}-T_{0}$ between adjacent BAC lines is less than two degrees, and the temperature drop $T_{0}-T_{\text {gas }}$ between the shield and bulk of the gas stream less than 0.2 degrees.

The system performance was calculated for a wide range of thermal boundary conditions (tank, environment, and cryocooler cold head temperatures) and total mass flow rates. Some of the results are plotted in Figs. 5 through 9 for the extreme case of $T_{\text {tank }}=20 \mathrm{~K}$ and $T_{e n v}=320 \mathrm{~K}$. In a few words, these results may be summarized thus: (1) For fixed inlet-to-outlet temperature rise, the net heat load on the shield decreases as the temperature of the shield increases; (2) For fixed temperature rise, the total required mass flow rate decreases as the shield temperature increases; (3) For fixed temperature rise, the magnitude of the inlet-tooutlet pressure drop (usually) increases as the shield temperature increases; (4) For fixed shield temperature, the required mass flow rate increases dramatically as the maximum allowable inlet-to-outlet temperature rise is constrained toward zero; and, (5) For fixed shield temperature, the inlet-to-outlet pressure drop increases dramatically as the maximum allowable temperature rise is constrained toward zero.

\section{Temperature Uniformity and Control}

It was assumed in the preceding section that the net heat flux on the shield is uniform, and therefore that all BAC lines encounter identical heat loads. It is perhaps not immediately obvious what effect a non-uniform heat load would have on the behavior of the network. For example, suppose that one line is loaded with a larger heat flux than the others. This means that the gas flowing in that line will, on average, be warmer. The viscosity of the gas increases with increasing temperature, so the warmer line will present a higher flow impedance, which means there will tend to be less mass flow in that line, which in turn means that it will be less efficient at removing heat. One might think that this process will lead to a runaway effect, that the warmer line will continue to become warmer still. But this line still sees the same pressure drop as the other lines, so there will be flow and therefore cooling. A new steady state must therefore necessarily be reached with the overloaded line at some higher average temperature than the others.

Consider, for example, five identical BAC loops with no thermal link between them, except at the inlet and outlet. Two limiting cases will be examined. The first case imposes a constant pressure drop across the network and allows the mass flow rate to vary. The second demands that the total mass flow rate remain constant, distributing 


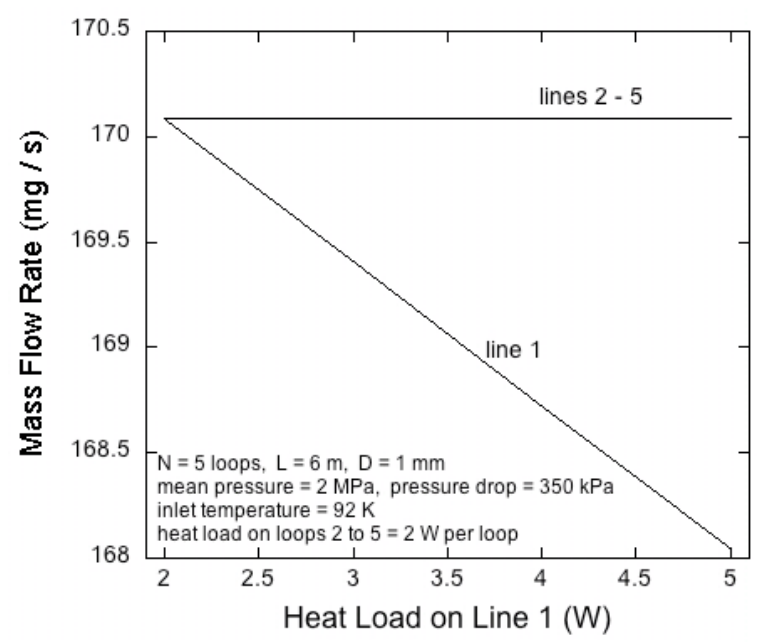

Figure 10. Effect of non-uniform heat load on mass flow rate in parallel BAC network (constant imposed pressure drop).

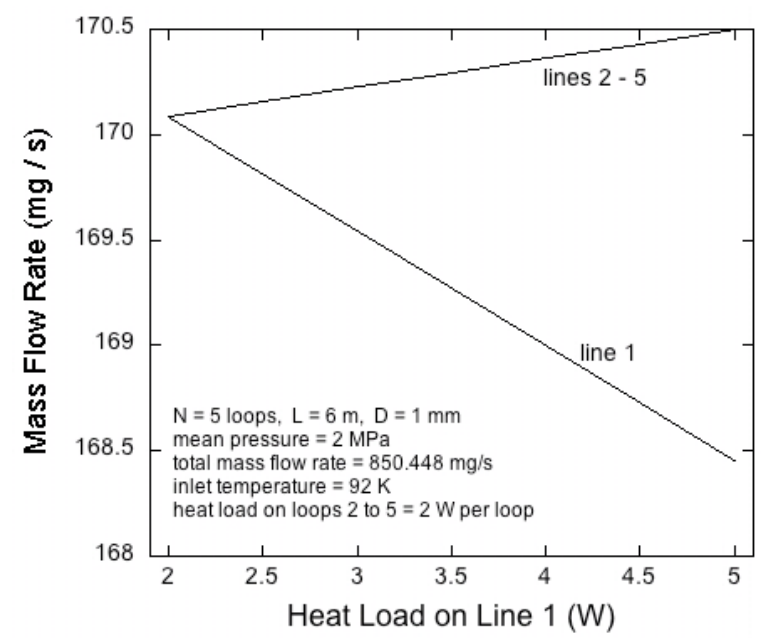

Figure 12. Effect of non-uniform heat load on mass flow rate in a parallel BAC network (constant imposed total mass flow rate).

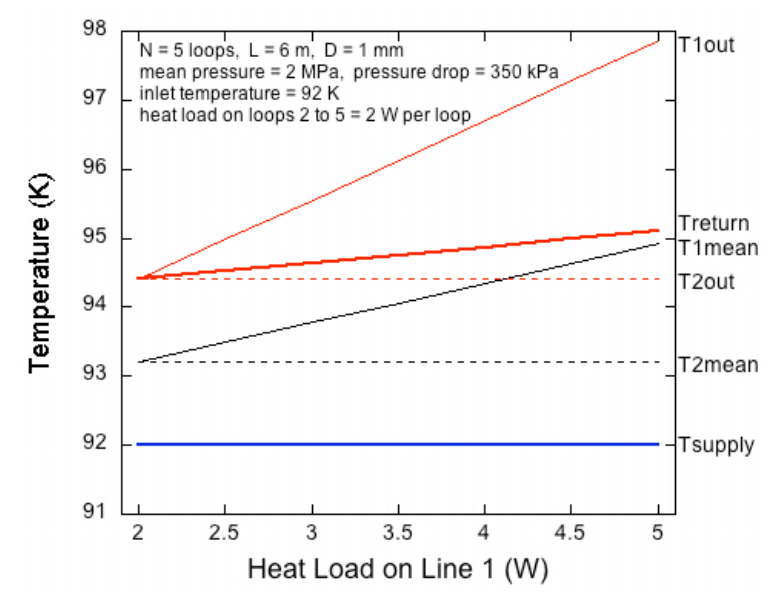

Figure 11. Effect of non-uniform heat load on BAC network temperatures (constant imposed pressure drop).

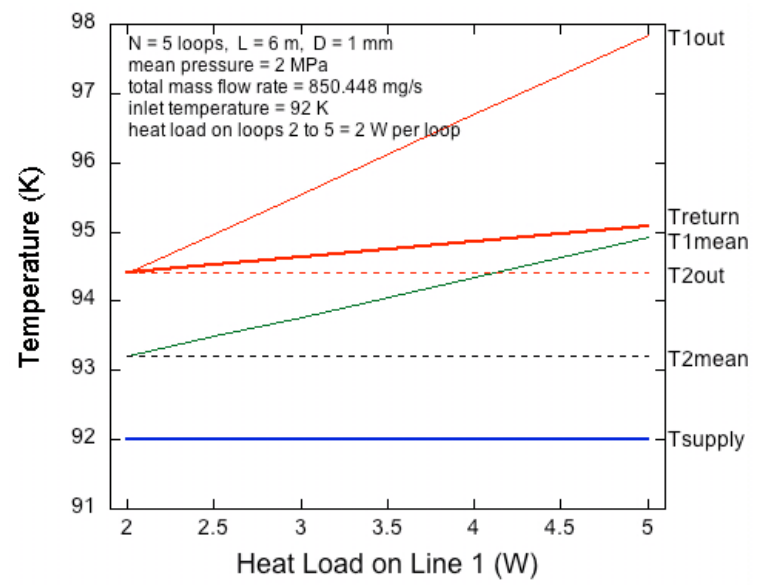

Figure 13. Effect of non-uniform heat load on BAC network temperatures (constant imposed total mass flow rate).

itself in such a way as to make the pressure drop the same in all lines. The actual result will be a sort of average of these two limits. Both calculations use the following numbers: line length $=6 \mathrm{~m}$; tube inner diameter $=1 \mathrm{~mm}$; inlet gas temperature $=92 \mathrm{~K}$; and the average pressure $=2 \mathrm{MPa}$. Suppose the heat load on each of lines 2 through 5 is 2 $\mathrm{W}$, and that the heat load on line 1 is varied from $2 \mathrm{~W}$ to $5 \mathrm{~W}$. The results of Case 1, with an imposed pressure drop of $350 \mathrm{kPa}$, are given in Figs. 10 and 11; and the results of Case 2, with an imposed total mass flow rate of 850.45 $\mathrm{mg} / \mathrm{s}$ (to match the Case 1 calculation when the heat load on line one is equal to $2 \mathrm{~W}$ ), are given in Figs. 12 and 13.

Both cases result in approximately the same mean temperatures and temperature rises. Even if the heat load on line 1 is more than doubled, its maximum temperature deviation, compared with the other lines, is only a few degrees. The system, while not strictly self-regulating, is nevertheless ultimately stable.

If non-uniform heat fluxes are anticipated, and if a stricter uniformity of temperature is required, more appropriate BAC geometries could be designed. For example, each line could be coiled around the tank (or other structure), so that all lines would see more or less the same total heat load. However, for precise temperature control of a multi-branched distributed cooling network within a thermal environment that varies in space or (especially) in time, an active flow control valve is required. Such a device is described in the next section. 


\section{Description of the Micro-Valve}

The technical requirements of the flow control valves are stringent. In order for a large number of cooling branches to provide controlled distributed cooling over large structures or arrays without significantly affecting the total size and mass of the system, it is desirable that the dimensions of the valves approach MEMS scale. In addition, they must be capable of a high degree of flow modulation, at least an order of magnitude. This will allow the system

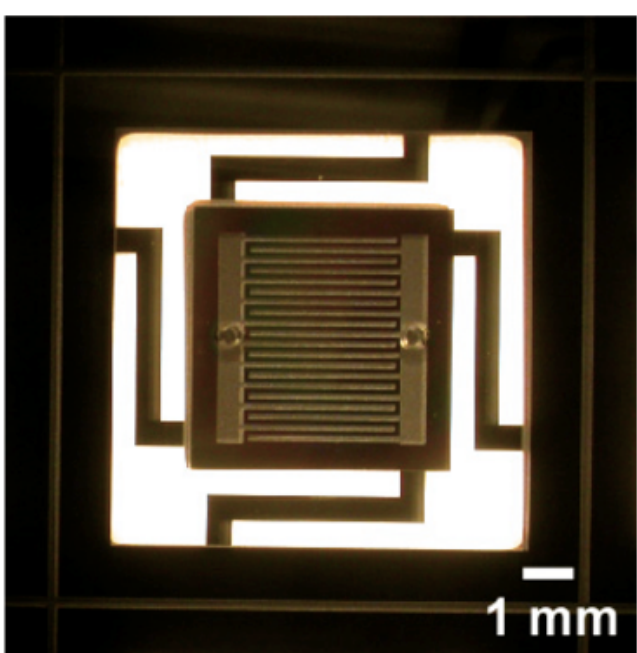

Figure 14. MEMS-patterned valve plate with flexures, fabricated from a single Silicon wafer. to respond to a wide range of operating conditions, provide a concentration of cooling power for quick response to varying thermal loads, and to reduce the cooling power essentially to zero within deactivated portions of the system. Finally, these valves must function reliably at high charge pressures (up to $\sim 2$ $\mathrm{MPa})$, and at low temperatures $(10$ to $100 \mathrm{~K})$ with very low power dissipation, so as to be compatible with the cryogenic systems in which they will be installed.

To address these requirements, researchers at UMSSEL and UWCEG, supported by Atlas Scientific and ARC, designed and modeled a piezoelectrically actuated micro-valve. Several prototypes have been fabricated and tested. The design ${ }^{8,9,10}$ consists of a series of shallow channels micro-machined into a Silicon substrate that seats against a Pyrex ${ }^{\circledR}$ plate. The channels form an inter-digital pattern (Fig. 14), communicating alternately with high- and low-pressure manifolds (the valve's inlet and outlet). When opened, gas flows through the channels and over the intervening lands. The valve conductance is approximately proportional to the separation between the patterned silicon plate and the Pyrex seat. Control of the valve is effected by energizing a stacked piezoelectric (PZT) actuator (Fig. 15).

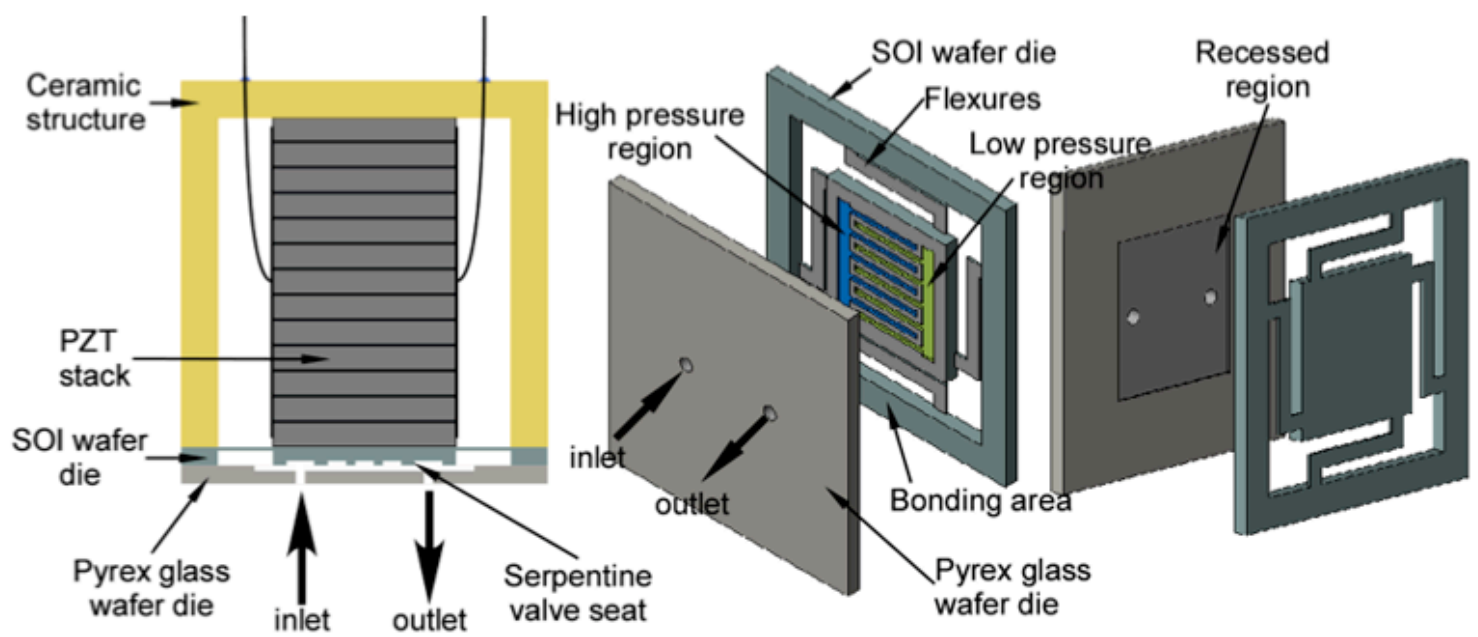

Figure 15. Left: Cross-section through a partially assembled micro-valve, showing the PZT actuator, MEMS-patterned valve plate / flexures, and the Pyrex valve seat. Center and Right: Bottom and top views, respectively, of the valve seat and the MEMS-patterned valve plate.

Because piezoelectric actuators are capable of applying large forces, and the effects of friction within the actuation mechanism are negligible, the valve can easily be actuated against a high differential pressure. A high actuation voltage $(\sim 100 \mathrm{~V})$ is required, but almost no current is drawn. The power dissipated when energized is on the order of a microwatt, and the control leads can be quite fine. The resulting heat leak is therefore minimal. However, the maximum displacement of the PZT stack is only a few microns at room temperature, and decreases as 


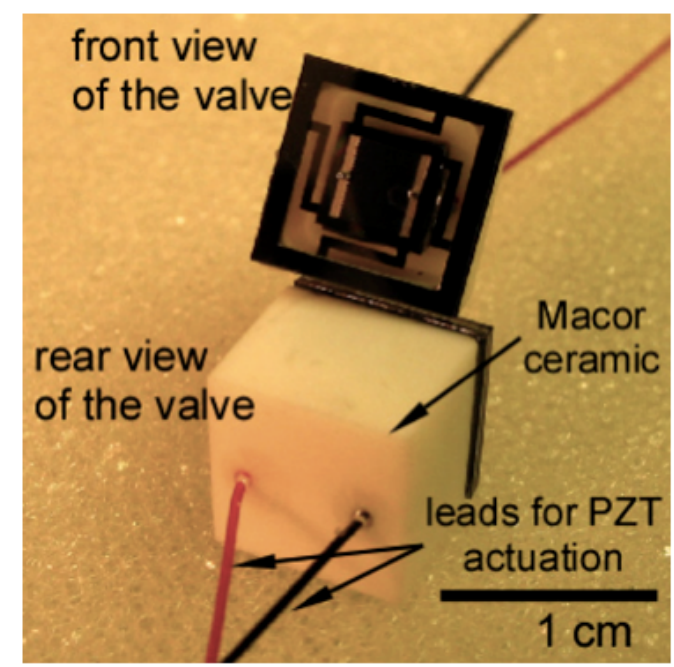

Figure 16. A prototype micro-valve before final assembly. the device is cooled. Because the achievable flow control is proportional to the maximum displacement, it is necessary to enhance the effective flow cross-section. This enhancement is provided by the micro-channels.

The structural body of the valve is fabricated from the machinable ceramic Macor ${ }^{\circledR}$, which has a coefficient of thermal expansion similar to that of the PZT stack. The inter-digital micro-channels were patterned by means of deep reactive ion etching 9 . The patterned Silicon wafers were then joined with a Pyrex substrate by anodic bonding. This process results in a consistent and precise geometry. The Silicon and Pyrex assembly was then bonded to the Macor cap using Sycast ${ }^{\circledR} 2850$ FT epoxy in a manual assembly process. Figure 16 shows one of the prototypes before the final assembly.

Room temperature measurements, with inlet pressures up to $100 \mathrm{kPa}$ and the exit at one atmosphere, indicate acceptable flow rates with exceptional modulation capabilities. Measurements at cryogenic temperatures and higher pressures are in progress.

\section{Acknowledgments}

The authors would like to thank NASA and the Missile Defense Agency for their support. The micro-valve development, analysis, and testing was primarily performed by the following individuals: Jong Moon Park and Allan Evans of the Solid State Engineering Laboratory at the University of Michigan-Ann Arbor; and Tyler Brosten, Ryan Taylor, Kris Rasmussen, Harrison Skye, Dan Hoch, and Prof. Sanford Klein of the Cryogenic Engineering Group of the University of Wisconsin-Madison.

\section{References}

${ }^{1}$ Christie, R., "Thermal Analysis of In-Space Cryogenic Propellant Depot with Conduction Shield Cooling," NASA ISCPD Project Internal Report, 2005.

${ }^{2}$ Feller, J. R., Kashani, A., Helvensteijn, B. P. M., Salerno, L. J., Kittel, P., Plachta, D., Christie, R., and Carlberg, E., "Analysis of Continuous Heat Exchangers for Cryogenic Boil-Off Reduction," Advances in Cryogenic Engineering, Vol. 52, 2007.

${ }^{3}$ Kashani, A., Maddocks, J. R., Nellis, G. F., and Gianchandani, Y, "Technologies for Cooling of Large Distributed and Deployable Loads," 2007.

${ }^{4}$ Diab, A. K., Nellis, G. F., Maddocks, J. R., and Yarbrough, S., "Development of a Rectifying Interface for a Hybrid PulseTube/Reverse-Brayton Cryocooler," Advances in Cryogenic Engineering, Vol. 49B, pp. 1777-1782, 2004.

${ }^{5}$ Creare, et. al.

${ }^{6}$ Feller, J. R., and Salerno, L. J., "Summary of Cryogenic Propellant Depot Test Bed Design Concepts," NASA ISCPD Project Internal Report, 2006.

${ }^{7}$ Feller, J. R., Salerno, L. J., Kashani, A., Maddocks, J. R., Helvensteijn, B. P. M., Nellis, G. F., and Gianchandani, Y. B., "Distributed Cooling Technologies for Cryogenic Boil-Off Reduction Systems," Proceedings, International Cryocooler Conference ICC-2008, to be published.

${ }^{8}$ Taylor, R., M. S. Thesis, University of Wisconsin-Madison, Mechanical Engineering Department, 2005.

${ }^{9}$ Park, J. M., Taylor, R. P., Evans, A. T., Brosten, T. R., Nellis, G. F., Klein, S. A., Feller, J. R., Salerno, L., and Gianchandani, Y. B., "A Piezoelectric Microvalve for Cryogenic Applications," IOP Journal for Micromechanics and Microengineering, Vol. 18, 2008.

${ }^{10}$ Brosten, T. R., "Model and Test of an Actively Controlled Cryogenic Micro-Valve," M. S. Thesis, University of Wisconsin-Madison, Mechanical Engineering Department, 2006. 